\title{
Tidal Data Generation for Sparse Data Regions in Han River Estuary Located in the Trans-Boundary of North and South Korea
}

\author{
Lian Guey LER ${ }^{1)}$, Klaus-Peter HOLZ ${ }^{2)}$, Gye Woon $\mathrm{CHOI}^{3)}$ and Seong Joon \\ $\mathrm{BYEON}^{4)^{*}}$
}

1)PhD Candidate, Incheon National University, lianguey@gmail.com 2) Professor Emeritus, Brandenburg University of Technology, kpeter.holz@hydroinf.com

3)Professor, Incheon National University, gyewoon@incheon.ac.kr

4)PhD Candidate, Incheon National University, seongjune@paran.com

* Incheon National University, Room 104, 7-46, Songdo-dong, Yeonsu-gu, Incheon, Korea <406-840>, E-Mail: seongjune@paran.com, Tel: +8232-850-5720, Fax: +82-32-851-5730 (Corresponding Author)

\begin{abstract}
The Han River estuary lies in the trans-boundary region of the North and South Korea. Thus as a result, it is often difficult to obtain continuous tidal data spanning over a long period of time. Thus, this paper proposed to set up a 2D Mike21FM numerical model to obtain accurate tidal level predictions of the Han River estuary in the Korean Demilitarized Zone. In order to obtain a good calibrated model, the location, shape and tidal stations' influence of the open sea boundary is extensively examined. In addition, a varying roughness coefficient based on the bathymetry's depth is used instead of a constant value for all depths. It is suggested that for future research, the model can be made more robust through further calibration using tidal forecasts as the input data for open sea boundary. This will eliminate the need for measured data for the open sea boundary in order to forcast accurate inland's stations levels.
\end{abstract}

Keywords: Han River Estuary, Mike21FM, Numerical Modelling, Tidal Level

\section{Introduction}

The Han River is the largest river of South Korea. It passes through the capital Seoul, flows through the demilitarized zone between the North and South Korea and finally ends as a wide estuary with extended tidal flats in Gyeonggi Bay. The city of Seoul and neighboring places are developing rapidly and thus intensive dvelopment projects are done on the river. For this good knowledge about the river dynamics and ecology is needed. However, because of downstream zones of no-entry between the countries field works are not possible, sparse data are available only and models operated are based on assumptions about downstream bathymetry and dynamics. This situation might be improved by having numerical models at hand simulating the downstream reach of the Han River and the Gyeonggi Bay.

The Gyeonggi Bay is characterized by extensive tidal shallows separated by mainly 3 deep tidal channels and some islands [1-3]. The average tidal range at the islands is about 4-5meters (macro-tidal), during spring and neap tides, the Gyeonggi Bay experiences a tidal range of $8 \mathrm{~m}$ and $3.5 \mathrm{~m}$ at coastal locations. The influence of the tides reaches up the Han river to locations near Seoul. In this reach the Han River has 2 tributaries which are the Imjin River and the YeSeong River originating in North Korea. 
The paper deals with building a numerical model for engineeing works covering this area. DHI software MKE21 version 2007 is use to solve the 2-D vertically integrated shallow water differential equations [4-6]. The discretization in the solution domain is performed using a finite volume method by subdivision of the continuum into nonoverlapping cells.

The model is driven by observed water-levels at 2 coastal stations. Using obseravtions is favourable as they account for all kind of secondary effects such as wind etc., with the option for the on-line use of the model. The discharge statistics of the Han River and its tributaries is used for specification of discharge boundary conditions. The approach differs from others where either oceanogrphic models / field measurments of the Yellow Sea or just theoretical tidal constituents have been used [7-10].

\section{Materials and Methods}

The model bathymetry was derived from multiple sources. The majority of the bathymetry data comes from the British Oceanographic Data Centre with a dimension of 900 x 900 [m] grid and lowest astronomical tide LAT as the datum. Released in September 2010 (prior to the construction of the Incheon Airport), the data is generated by combining quality-controlled ship depth soundings with interpolation between sounding points guided by satellite derived gravity data. In some areas, especially the downstream of the Han River where the shallow banks and mudflats are located, the data from the BODC do not provide sufficient details. Hence, additional survey measurement data of the along the Han River at 500 [m] interval and satalite data of the Han River mouth is added to the bathymetry database. The mesh for the model is generated based on the bathymetric information, where the mesh size is adjusted manually in areas with sudden changes like banks, channels and narrows. The number of nodes implemented in the model is around 3500 with more than 5500 elements created.

\section{Open Boundaries and Model Extend}

Open boundaries are commonly put at locations of the tidal gauging stations. In the first model set-up, the open sea boundary is placed between DaeYeunPyongDo, DukJukDo and DaeSan (Table 1 - Boundary 1). However, this was found to result in poor correlation at nearby tidal locations (AnSan, Incheon and PyeongTeak). The reason might be due to the fact that 2 of the 3 stations were by correlation forecasted tidal data. Also, these stations are deemed too closed to the coastal stations where they will exert an unwanted significant influence on the inland stations. It was therefore decided to extend the model further outwards into the Yellow Sea, locating the boundary between DaeChongDo and AnHueng, encompassing the entire bay of the Han River Mouth.

Once the extend of the model has been decided, the next phase is to determine the shape of the open sea boundary. Based on the M2 cotidal map in a research conducted by Fang, et al., which is constructed from tidal gages and satellite observations, one can observed that the tidal waves propagate approximately parallel to the mean coastal line [11].

Table 1. List of Tidal Gauging Stations

\begin{tabular}{lllll}
\hline Type & Station Name & Lon & Lat & Type \\
\hline \multirow{2}{*}{ Boundary 1 } & DaeSan & 37.00472 & 126.3544 & Measurement \\
& DaeYeunPyongDo & 37.66667 & 125.7167 & Predicted \\
& DukJukDo & 37.25 & 126.1333 & Predicted \\
\hline \multirow{3}{*}{ Boundary 2 } & AnHueng & 36.67 & 126.135 & Measurement \\
& DaeChongDo & 37.825 & 124.7181 & Measurement
\end{tabular}




\begin{tabular}{lllll} 
& AnSan & 37.02278 & 126.6497 & Measurement \\
Validation & Incheon & 37.44917 & 126.5942 & Measurement \\
\& Calibration & PyongTeak & 36.96389 & 126.8247 & Measurement \\
\hline
\end{tabular}

This observation is useful in a way, although it does not solve the problem of how to interpolate tidal data between the stations AnHung and TaehongDo (figure 1), at which the seaward boundary has to be specified. As the high water level and phase differ between both stations a try-and-error procudure was applied to find the best boundary parameters in terms of location (shape) and interpolation.

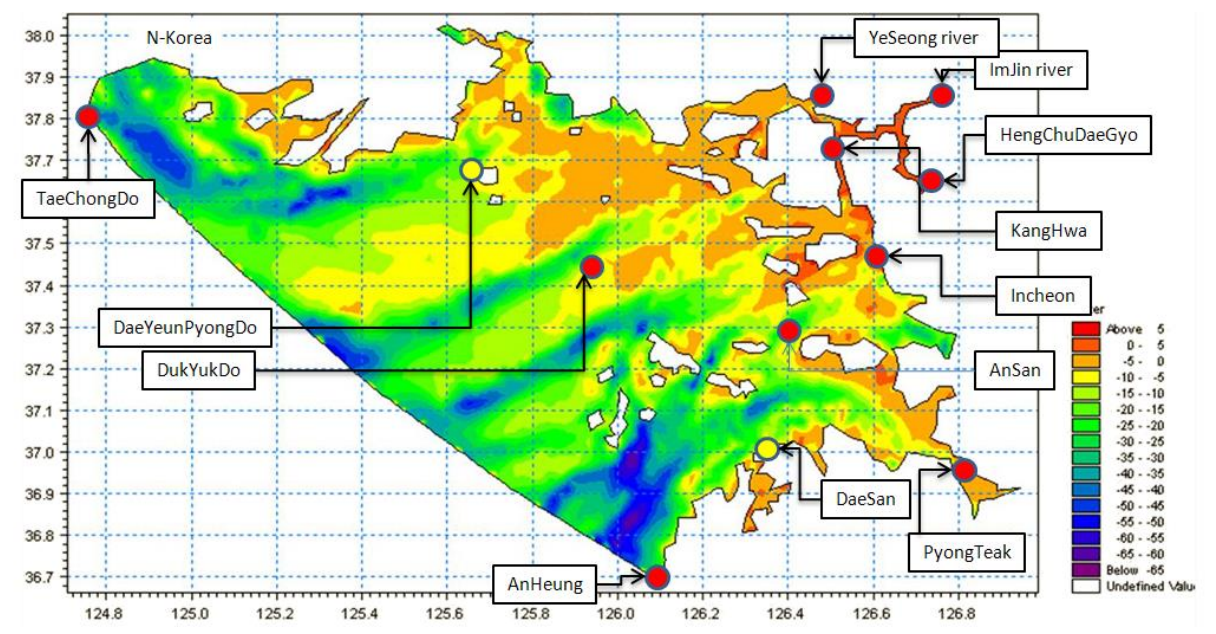

Figure 1. Locations of Tidal Gauging Stations

Models with different shapes for the open sea boundary were setup as shown in Figure 2. They were validated with observed tidal data at coastal stations. It should be noted that the boundary should be able to include the deep water zones in front of the tidal channels or across them. The different configurations are shown in Figure 2. It is found that the more concave the open sea boundary is chosen the bigger positive phase error are. When the open sea boundary is straight, the phase error becomes negative in value due to the shorter distance from the coast. It was felt that this might be compensated by the nonlinear interpolation of the tidal water-level along the boundary btween AnHung and TaehongDo. 

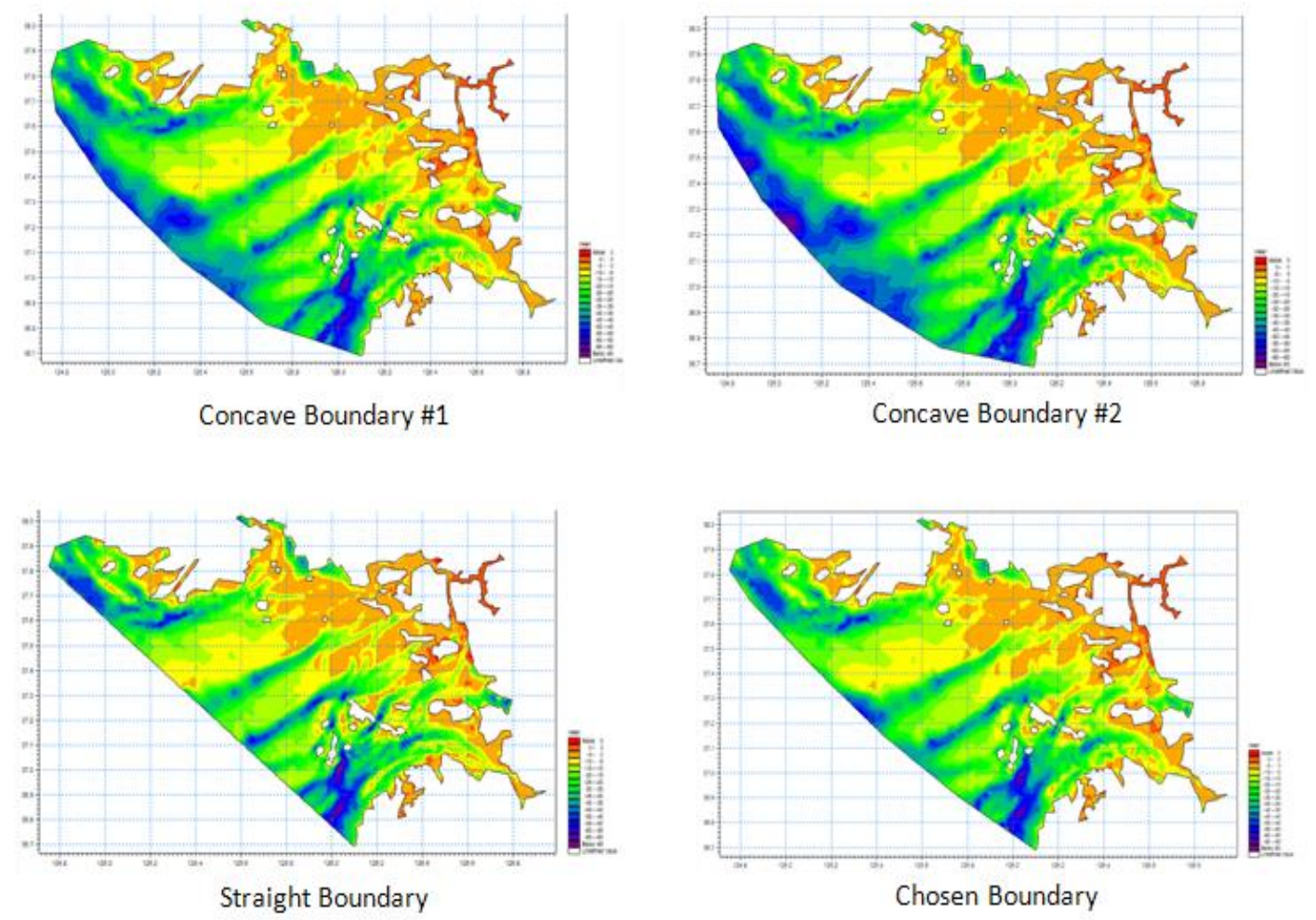

Figure 2. Different Shapes of the Open Sea Boundary

Such a configuration gives a reasonably good representation for AnSan coastal station but poor representation for Incheon and GangHwa gauges when compared to the observation data. The predictions from the model constantly give a positive phase error as well as poor low water agreement.

The considerations of "watersheds" and "tidal channels" is essential for the understanding the mechanism of tidal propagation in the shallow highly structured intertidal water. According to a study by Woo S.B. et al., the tidal wave propagates through the Yeumha, Seokmo and Kyodong channels [3]. As most of the tidal energy comes from the tidal waves travelling through these deep channels, it is evident that AnHeung tidal gauging station located near the Yeumha channel exert a much more significant influence than DaeChongDo tidal gauging station. Therefore, instead of using the linear interpolation of the stations, the open sea boundary is split into 3 sections, with the first section corresponding to the DaeChongDo tidal gauging station, the last section corresponding to the AnHeung tidal gauging station and the middle section corresponding to the linear interpolation of the 2 stations.
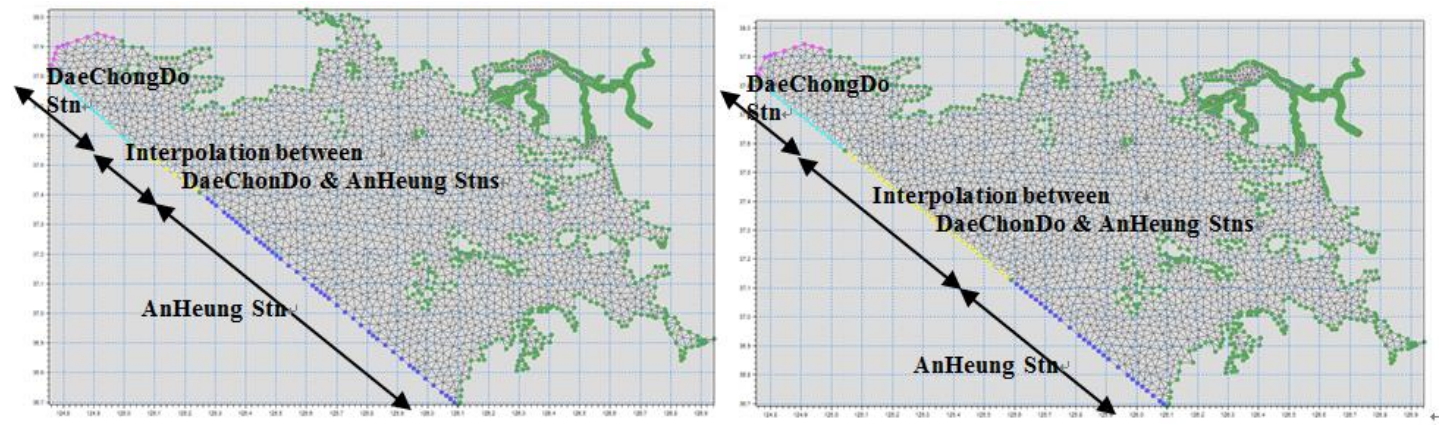


\section{Figure 3. Bathymetry File Showing the Different Distribution of Tidal Gauging Stations' Influence across the Open Sea Boundary}

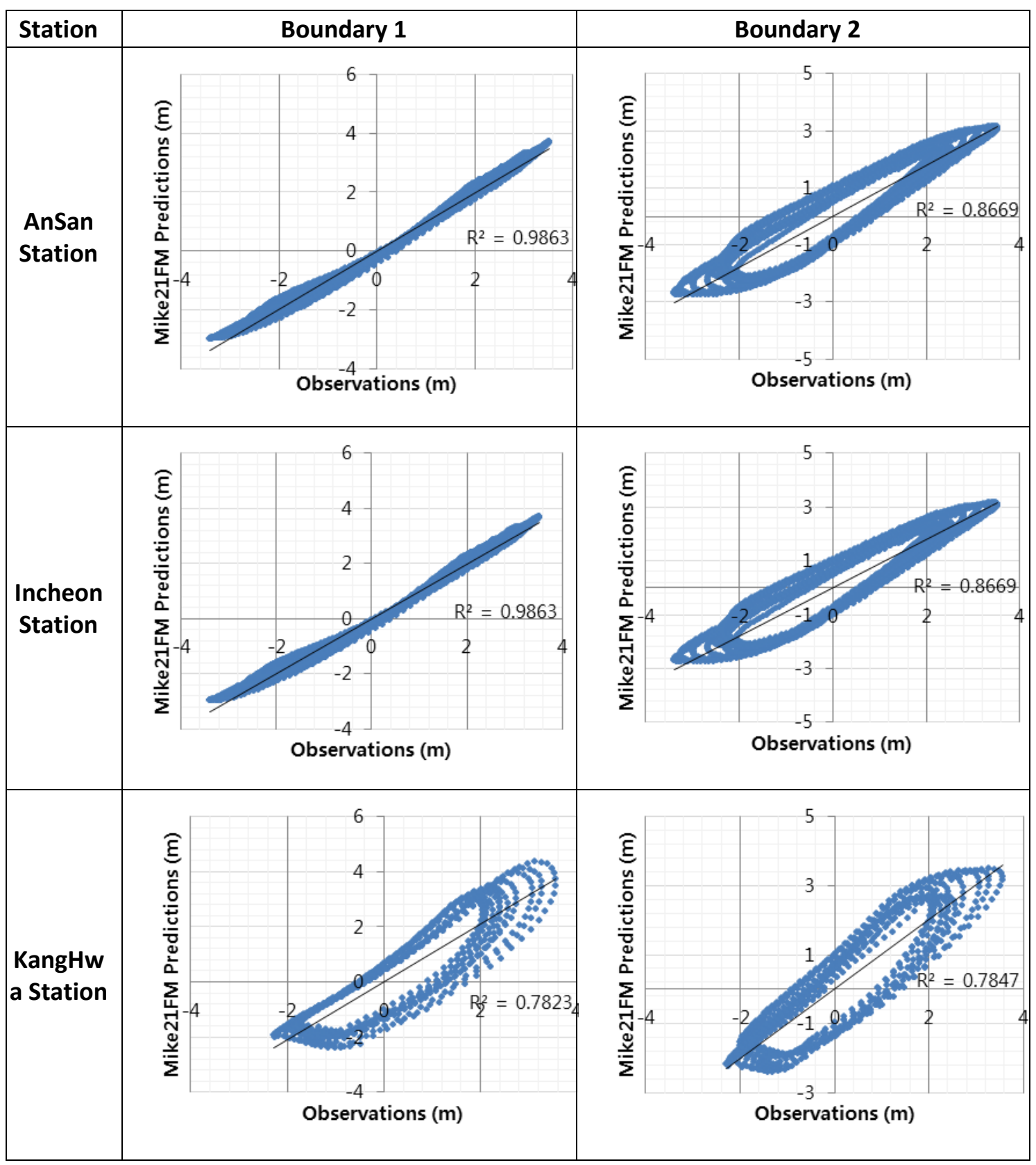

Figure 3. Plots of Tidal Observations against Mike21FM Predictions

Figure 3 shows two different configuration of the tidal gauging stations' distribution; with one (Boundary 1) having direct influence from the AnHeung station for about twothirds of the open sea boundary while the other (Boundary 2) having only one-third direct influence from the AnHeung station

This configuration was found to be the best fit where more detailed simulations were run using this configuration. Frist it was found that the specification of any discharge in the Han River has no influence on the tidal signals at AnSan and Incheon. However it was implemented because of the significant influence on the GangHwa statio, which is located close to the Han River mouth. This is shown by applying discharge data from the 3 
available gauging stations at the Han River (HengChuDaeGyo), the Yesong River and the Imjin River as upstream boundary conditon.

For the validation of the simulations, data for the sea tidal gauging stations have been used. They stem from the Korea Hydrographic and Oceanographic Administration (www.khoa.go.kr) while the data for the river gauging stations comes from the Han River Flood Control Office (www.hrfco.go.kr) [12].

Simulations were run for this configration of the model with the observation data and boundary interpolations while keeping the friction and viscosity parameters unchanged and constant in space and time. The results show that when AnHeung is given a direct influence over two-thirds of the boundary, it gives a significant improvement in the predictions of the coastal stations, especially in Incheon station and AnSan station as shown in Figure 4. However, for GangHwa station, there is no significant improvement in the predictions.

\section{Model Calibration and Validation}

The model of boundary interpolation type 1 (AnHeung dominates $2 / 3$ of the boudary) was calibrated against the 3 coastal tidal gauging stations: Incheon, AnSan and GangHwa. The observation data for the tidal gauging stations come from the Korea Hydrographic and Oceanographic Administration (www.khoa.go.kr), measured in 1 minutes interval for Incheon and AnSan and 10 minutes interval for GangHwa [12].

In the prior calibrations, the roughness coefficient for the bathymetry has been set as a constant value for all depths. And it is deduced that because of this, it led to the poor model predictions for the shallow waters in KangHwa station. Therefore, a varying roughness coefficient is assigned according to the element's bathymetry depth as shown in Figure 5 below.

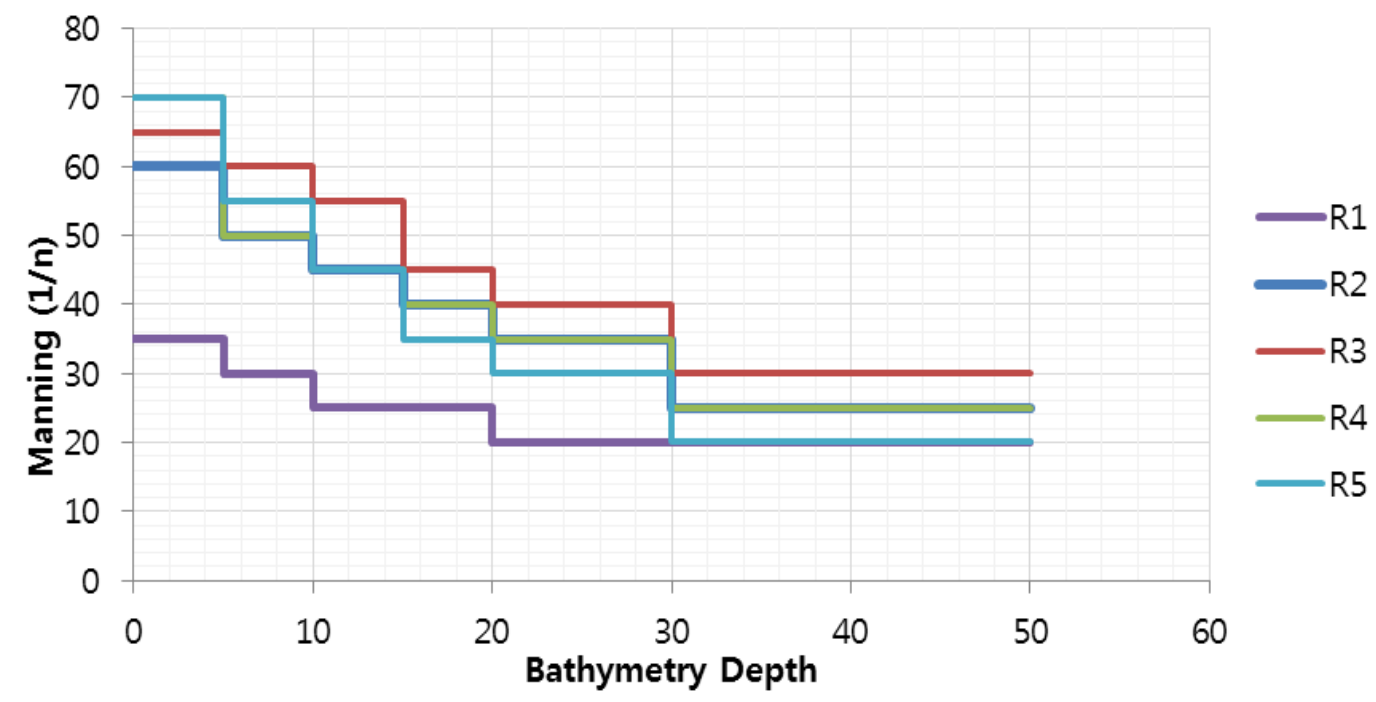

\section{Figure 5. Variation in the Manning Number for Varying Bathymetry Depth}

It should be noted that the resistance values used should not be treated as the real roughness manning coefficients of the seabed bathymetry. This is because in this calibration, the eddy current coefficient is kept constant at 0.4 . Thus, the resistance values used here should be treated as merely a calibration parameter.

The range of the manning number (R1 and R2) for the varying bathymetry depth is based on values from David L. [13]. The chosen values for R1 corresponds to a region dominated by smoother (flatter) beds while R2 represents a highly energetic channel region. R3, R4 and R5 are ranges which are composites of R1 and R2. Among the five different ranges, R5 provides the best predictions for the coastal stations, as shown in 
Figure 5. Once the resistance coefficients has been determined, the model is run for over a month in order to verify that it is able to accurately simulate the full 28 days of the lunar cycle. The time series plots are shown in Figure 6. As evident, the amplitude difference for both AnSan and Incheon stations are within 5 to $15 \mathrm{~cm}$ while that of KangHwa station is within 25 to $50 \mathrm{~cm}$.

\section{Tidal Data Generation for Sparse Data Regions}

To validate that the simulated tides in the Mike21FM model is behaving correctly, a co-tidal map is plotted from the simulated results (Figure 6) and compared with the M2 co-tidal map (Figure 7) constructed from the tide gauge and satellite observations by previous study [8].

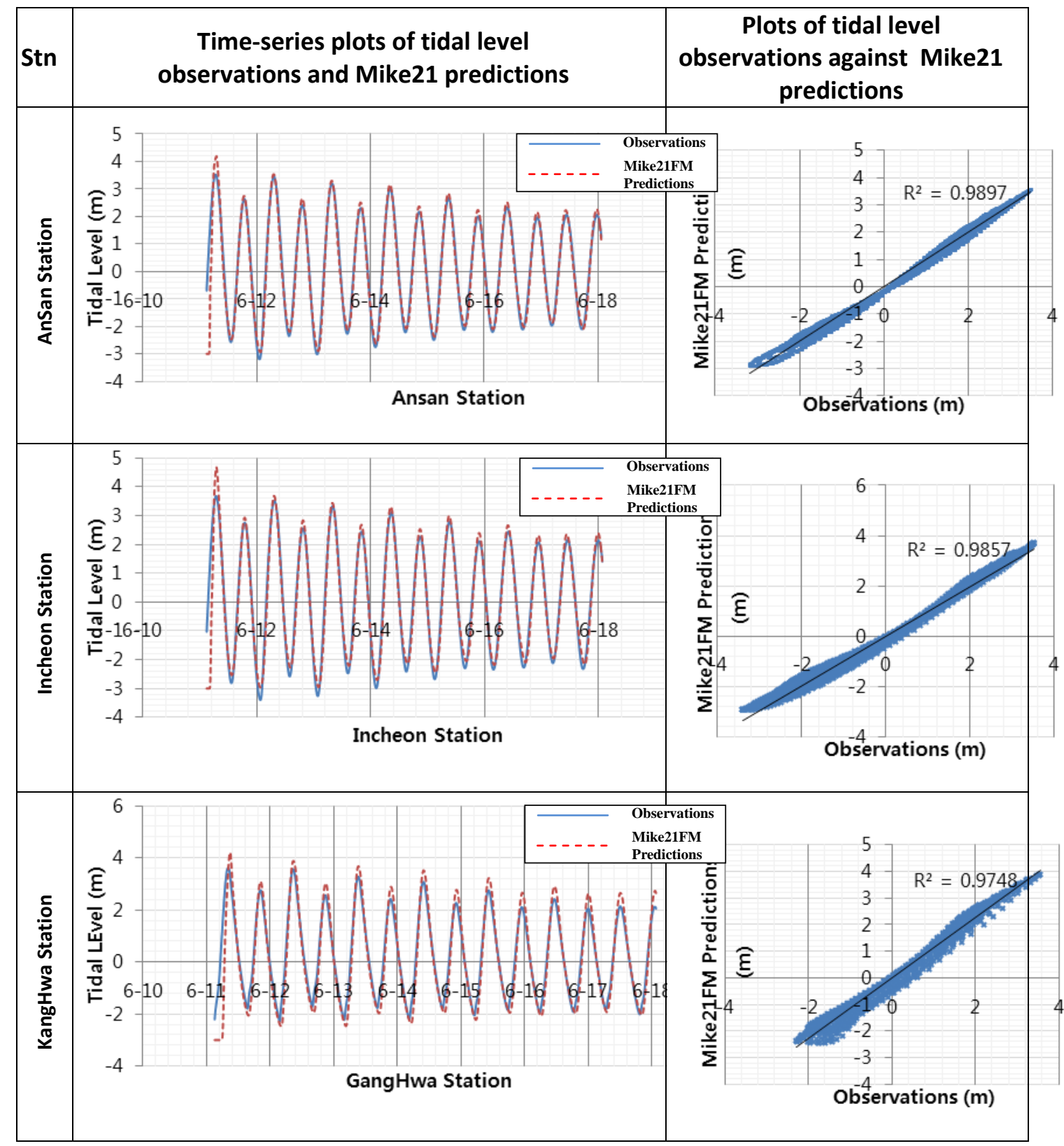

Figure 6. Plots of Tidal Level Observations and Mike21FM Predictions 

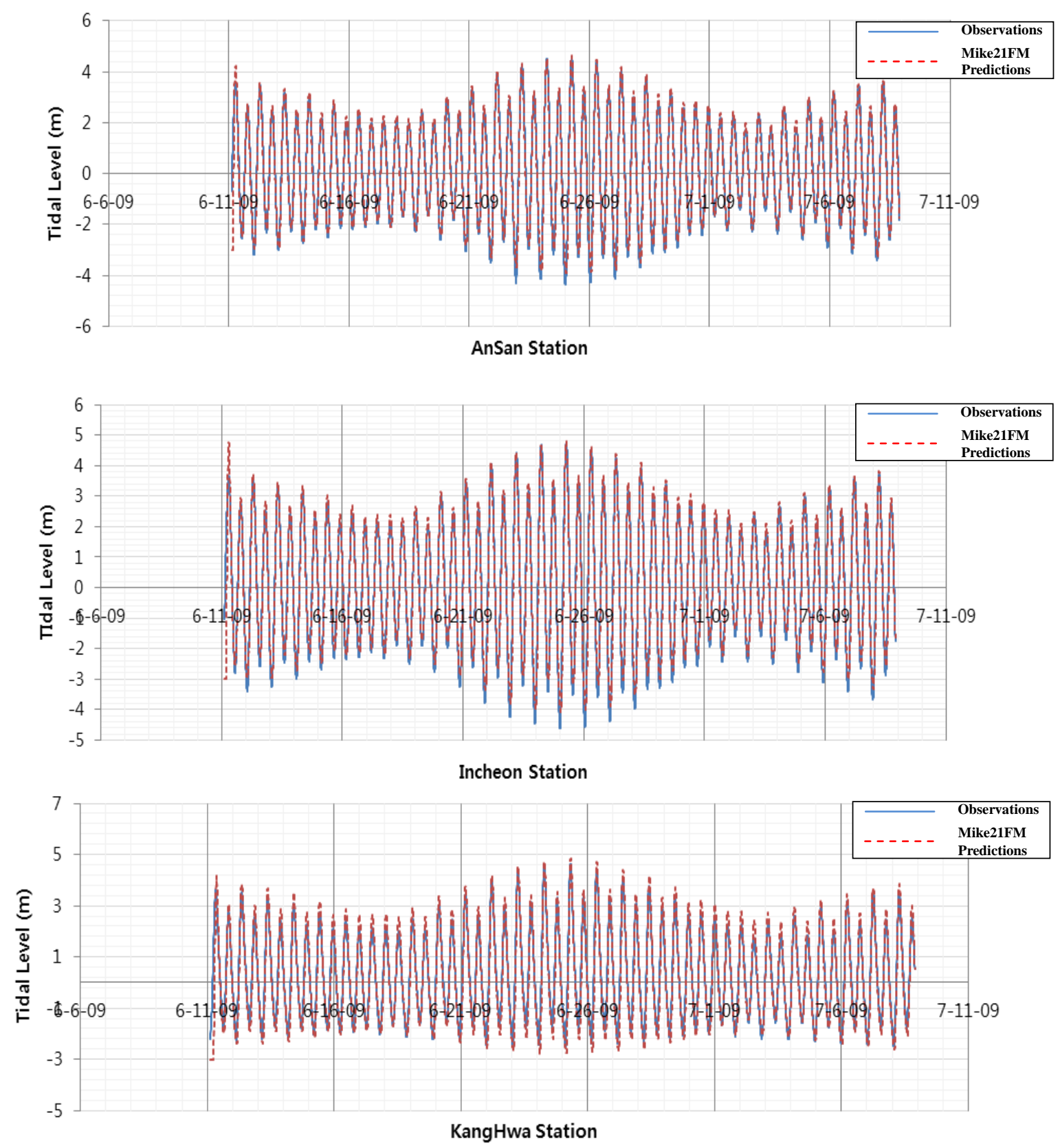

Figure 7. Plots of Tidal Level Observations and Mike21FM Predictions for 1 Month

As evident in Figure 8 and Figure 9, the Mike21FM simulated waves are propagating in a similar manner with similar tidal amplitudes of the M2 co-tidal waves. There is a good agreement from the southern part of the bay, while the northern part differs slightly. This is probably due to the small bays located near the TaeChongDo station and the middle top of the boundary, where the localized eddy currents are accounted in the Mike21FM model, unlike in the M2 tides. 


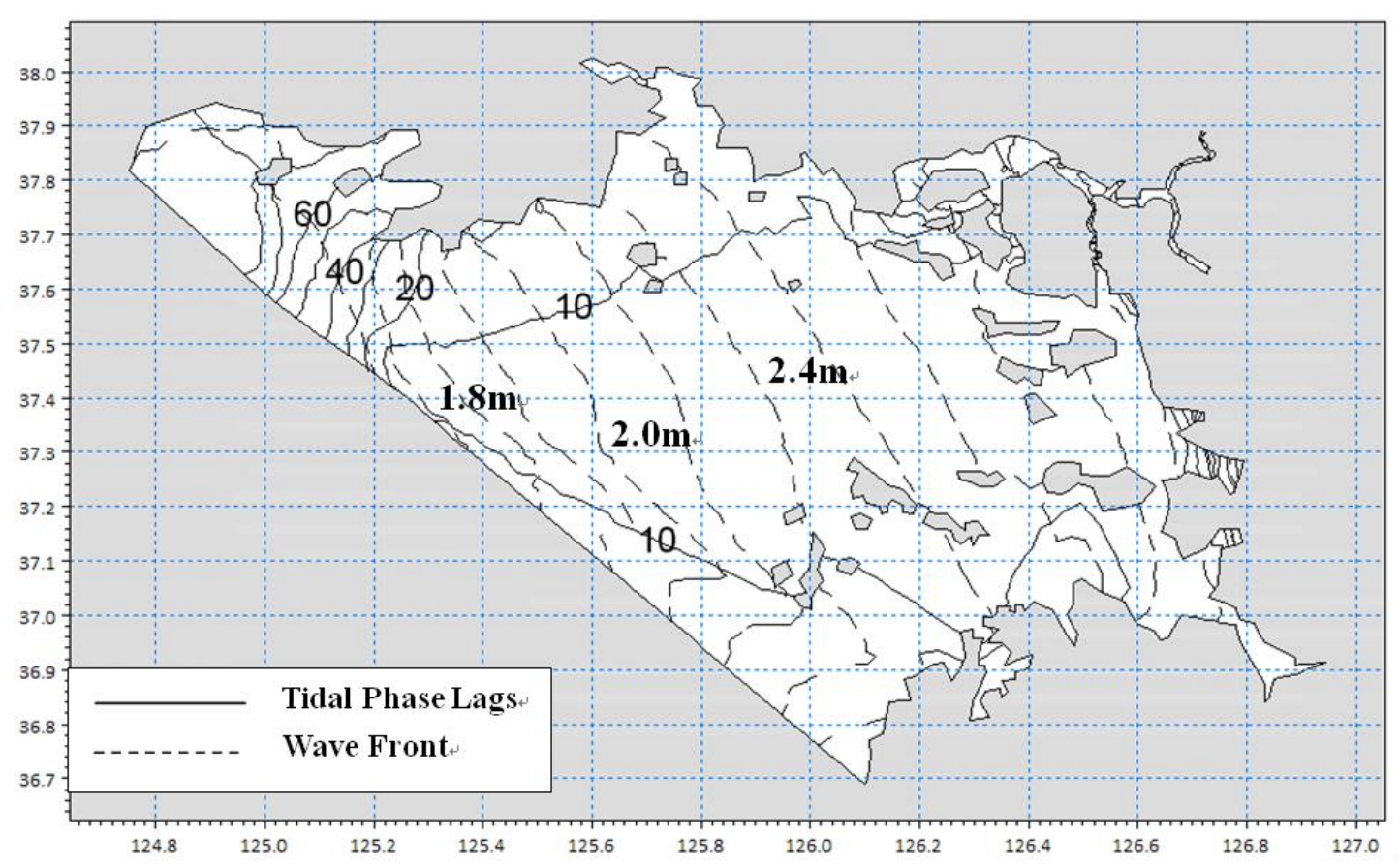

Figure 8. Tidal Map of Mike21FM Model Results

(Tidal Phase Lag of 10 Minutes Interval, Tidal Amplitudes of 0.2 Meters Interval)

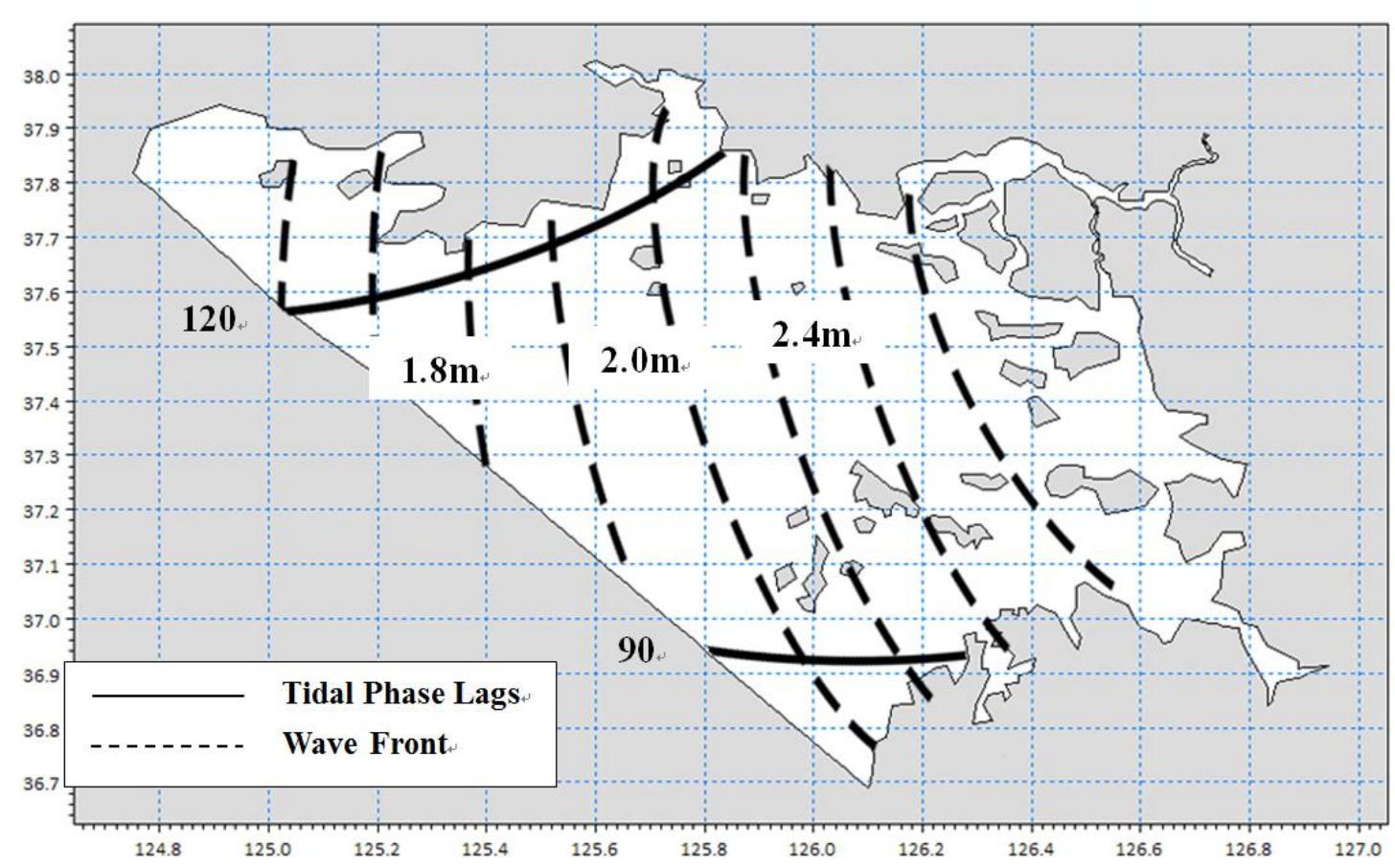

Figure 9. Tidal Map of M2 Tides Constructed from Research by Fang, et al., (2004) (Tidal Phase Lag of 30 Degree Interval, Tidal Amplitudes of 0.2 Meters Interval) 


\section{Conclusions}

As the Han River estuary is partially located in the Korean Demilitarized Zone, there is limited or in some areas totally no access to it. As a result, there is a lack of field measurements of the tidal conditions and bathymetry for the Han River estuary in that region. Therefore, it is of this concern that this research aims to generate tidal condition data in those sparse data regions.

A one year dataset of tidal level for setting up a numerical model for that area was collected. The measurements were compared with the results from a 2D hydrodynamic model using MIKE21 FM software. Since the boundary is positioned far seawards from the Han River estuary, the winds effects are ignored in the numerical modeling.

Calibration was carried out by considering the boundary properties such as the location, shape and distribution of the tidal stations' influence as well as the roughness coefficient in relation to the bathymetry depth. Below are the summary of the calibration process results:

1. The boundary of the model is placed far away from the Han River estuary in the deep waters region to avoid local effects of tidal channels

2. As there are only 2 coastal tidal gauging stations located at the ends of the boundary, there is a need to determine an appropriate interpolation along the seaward boundary reflecting the influence of the 2 tidal stations across the whole boundary. It is found that AnHeung station has a dominant influence on the tidal conditions in the Han River estuary. This is also verified by previous researches, where 2 of the 3 tidal channels are influenced by the AnHeung station.

3. Varying roughness coefficients are used for different bathymetry depths to obtain better results in the Han River estuary. It should be noted that the determined roughness coefficients obtained should not be taken as a real representative of the bathymetry roughness, as a constant eddy force instead of a varying one is used in this model.

4. A tidal map is plotted from the calibrated model results and compared with the M2 co-tidal map constructed from the tide gauge data and satellite observations by (Fang, et al., 2004). Generally there is a fairly good agreement with the tidal amplitudes and phase lag, with the exception in the northern part of the model. It is believed this is caused by the small bays having local eddy currents and reflections. Proof cannot be given as the Northern Coast is a no-entry zone.

5. The calibrated model is able to produce predictions of accuracy within of $10 \mathrm{~cm}$ to $15 \mathrm{~cm}$ for the coastal stations of Incheon and AnSan and $25 \mathrm{~cm}$ to $50 \mathrm{~cm}$ for GangHwa station inside the estuary.

In addition, the model can be made more robust through further calibration using tidal forecasts as input data for the open sea boundary. This will eliminate the need for measured data for the open sea boundary in order to forcast accurate inland's stations levels.

\section{ACKNOWLEDGEMENTS}

This research was funded by Incheon National University in 2010, South Korea, as part of the International Co-Operation Research program.

\section{REFERENCES}

[1]. H. S. Kim, "Numerical modeling of tidal dynamics and salinity distribution in Gyounggi Bay", MS Thesis, Inha University, Incheon, Korea. 61p. (in Korean with English abstract), (1997).

[2]. K. Park, J. H. Oh, H. S. Kim and H. H. Im, "Case study: Mass Transport Mechanism in Kyunggi Bay around Han River Mouth", Journal of Hydraulic Engineering, vol. 128, no. 3, (2002), pp. 257-267. 
[3]. S. B. Woo and B. I. Yoon, "The classification of estuary and tidal propagation characteristics in the Gyeong-Gi Bay, South Korea", Journal of Coastal Research, SI 64 (Proceedings of the 11th International Coastal Symposium), Szczecin, Poland, ISSN 0749-0208, (2011), pp. 1624-1628

[4]. "DHI, MIKE 21 \& Mike 3 Flow Model FM - Hydrodynamic Module User Guide", Danish Hydraulic Institute, (2007).

[5]. "DHI, MIKE 21 \& Mike 3 Flow Model FM - Hydrodynamic and Transport Module Scientific Documentation", Danish Hydraulic Institute, (2007).

[6]. S. J. Byeon, G. W. Choi and H. G. Jo, "A study on discharged coliform into coastal bathing water after rainfall event", Journal of Korean Society of Hazard Mitigation, in Korean, vol. 11, no. 5, (2011), pp. 337-343.

[7]. I. J. Moon, "Impact of a coupled ocean wave-tide-circulation system on coastal modeling", Ocean Modelling, ISSN 1463-5003, 10.1016/j.ocemod.2004.02.001, vol. 8, Issue 3, (2005), pp. 203-236.

[8]. W. J. Teague, H. T. Perkins, Z. R. Hallock and G. A. Jacobs, "Current and tide observations in the southern Yellow Sea”, J. Geophys. Res., 103(C12), doi:10.1029/98JC02672, (1998), pp. 27783-27793.

[9]. D. Bisht, S. Jain and M. M. Raju, "Prediction of water table elevation fluctuation through fuzzy logic and artificial neural networks", International Journal of Advanced Science and Technology, vol. 51, (2013), pp. 107-120.

[10].D. Datta and H. S. Kushwaha, "Uncertainty quantification using stochastic response surface method case study - transport of chemical contaminants through ground water", International Journal of Energy, Information and Communications, vol. 2, no. 3, (2011), pp. 49-58.

[11].G. Fang, Y. Wang, Z. Wei, B. H. Choi, X. Wang and J. Wang, "Empirical cotidal charts of the Bohai, Yellow, and East China Seas from 10 years of TOPEX/Poseidon altimetry", J Geophys Res Oceans, 109(C11):C11006, (2004)

[12]."MOLIT, Basic Han River Maintenance Plan Report : Paldang Dam - Han River Mouth, Ministry of Construction and Transportation", Seoul Regional Construction Management Office, No. 2003.30, 11 1500185-000022-14, in Korean, (2002).

[13].L. David, "A Review of the Bed Roughness Variable in MIKE 21 FLOW MODEL FM", Hydrodynamic (HD) and Sediment Transport (ST) modules, University of Southampton, UK, (2010).

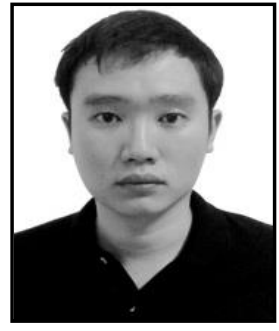

Lian Guey Ler Received his BSc degree in Civil Engineering in 2007 from National University of Singapore and his M.S. Degree in Hydro informatics (Engineering) in 2009 from University of Nice Sophia Antipolis. He is currently finished his Ph.D. coursework and preparing his dissertation to receive his Ph.D degree from Incheon National University. His research interests include numerical analysis and computer science to apply water management.

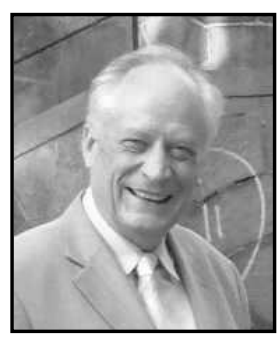

Klaus-Peter Holz Received his B.S. degree in Civil Engineering, his M.S. degree and his Ph.D. degree in Mechanics in Civil Engineering from University of Hannover, Germany, in 1965, 1970 and 1975 respectively. During 15 years (1980-1994), he had been a professor for Computational Civil Engineering at University of Hannover, Germany. And since 1994, he had been a faculty member at Brandenburg University of Technology, Cottbus, Germany until 2008. His main expertise/research interests are numerical modelling of free and subsurface flow and transport process by finite element method and artificial neural networks. 


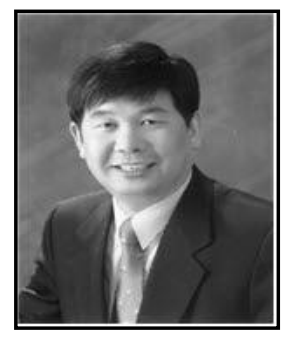

Gye Woon Choi Received his B.S. degree in Civil Engineering in 1982 from Inha University, Incheon, Korea and his M.S. Degree in Water Engineering in 1985 in Seoul National University, Seoul, Korea. And he received his Ph.D. degree in Water Engineering in 1991 from Colorado State University, Colorado, USA. Since 1994, he has been a faculty member at the Department of Civil and Environmental Engineering of Incheon National University, Incheon, Korea. His research interests include hydro informatics (convergence of water engineering, mathematics and computer science), hydraulics, and hydrodynamics.

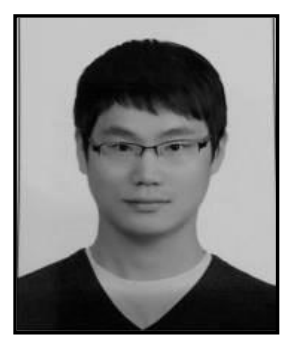

Seong Joon Byeon Received his BSc degree in Civil Engineering and his M.S. Degree in hydro informatics (Engineering) from Incheon National University, Incheon, Korea, in 2005 and 2008 respectively. And now he is Ph.D. candidate under the defense procedure to receive the Ph.D. degree in hydro informatics (Engineering) from Incheon National University, Incheon, Korea, and University of Nice Sophia Antipolis, Nice, France as dual degree program. His research interest is convergence of computer science and water engineering. 